\title{
Modélisation numérique du phénomène de fretting-fatigue intervenant dans le vieillissement des conducteurs de lignes aériennes
}

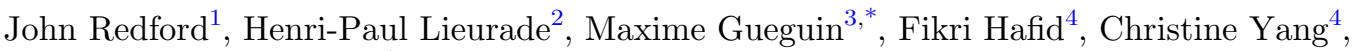 \\ et Jean-Michel Ghidaglia ${ }^{1}$ \\ ${ }^{1}$ CMLA, ENS Paris-Saclay, CNRS, Université Paris-Saclay, 94230 Cachan, France \\ ${ }^{2}$ HPL Consulting, 4 rue du Fer à Cheval, 78100 Saint-Germain-en-Laye, France \\ ${ }^{3}$ Eurobios, 61 avenue du Président Wilson, 94235 Cachan, France \\ ${ }^{4}$ RTE, Direction R\&D-I, 9 rue de la Porte de Buc, 78005 Versailles, France
}

Reçu le 2 février 2018 / Accepté le 12 juin 2018

\begin{abstract}
Résumé. Les travaux présentés dans cet article s'inscrivent dans un projet de R\&D mis en place par RTE (Réseau de Transport d'Electricité) et visant à évaluer le vieillissement des conducteurs électriques de lignes aériennes par des outils de simulation numérique. Après avoir rappelé la géométrie de tels conducteurs et l'utilisation qui en est faite, le principal risque d'endommagement de ces derniers est présenté. Il s'agit du phénomène de fretting-fatigue intervenant à proximité des systèmes de fixation des conducteurs au niveau des pylônes, pouvant produire la rupture de brins les constituant. Ainsi, une méthodologie originale est proposée de façon à évaluer, par l'intermédiaire d'un modèle éléments finis, la durée de vie des conducteurs vis-à-vis de ce risque. Cette méthodologie est ensuite appliquée sur un cas-test dont les données proviennent de la littérature. Enfin, des perspectives sont données concernant le modèle numérique employé et l'intégration de la démarche dans le projet de R\&D concerné.
\end{abstract}

Mots clés : lignes aériennes / conducteurs électriques / fretting-fatigue / modélisation éléments finis

\begin{abstract}
Numerical modeling of the contribution made by fretting-fatigue to aging of overhead line conductors. The work presented in this article is part of a R\&D project set up by RTE (Réseau de Transport d'Electricité) to evaluate the aging of electrical conductors in overhead lines using digital simulation tools. After giving some details on the conductors, including their geometry and how they are put to use, the principal damage risk is presented. This is the phenomenon of fretting-fatigue that occurs near the fixing systems that attach the conductors to the pylons, causing the rupture of the constituent strands. Thus, a finite element model is used as part of a proposed original methodology to evaluate conductor lifetime due to this risk. This methodology is then applied to a test-case from the literature. Finally, future plans are given concerning the numerical model and the integration of the approach into the $\mathrm{R} \& \mathrm{D}$ project.
\end{abstract}

Keywords: overhead lines / conductors / fretting-fatigue / finite element method

\section{Mise en contexte de l'étude du vieillissement des lignes aériennes du parc de RTE}

\subsection{Description du projet de recherche}

RTE a en charge la gestion du transport d'électricité hautetension sur l'ensemble du territoire métropolitain français. Parmi les technologies utilisées pour ce transport, les lignes

\footnotetext{
* e-mail: mgueguin@eurobios.com
}

aériennes en représentent une part importante, puisqu'elles constituent un parc de plus de 100000 kilomètres. Il est donc évident que le remplacement des conducteurs électriques qui les composent est un sujet majeur pour l'entreprise. À l'heure actuelle, RTE envisage le remplacement de ces matériels à partir de leurs 85 ans, quelle que soit leur position sur le territoire. Or, l'installation des lignes aériennes a connu un essor majeur entre 1950 et 1960. Continuer à baser sa politique de remplacement de conducteurs sur ce seuil subjectif de 85 ans impliquerait donc pour RTE des moyens humains et financiers importants dans les décennies à venir. Une remise en 
cause de cette règle interne est d'autant plus en question que, dans une part non négligeable des remplacements effectués au bout de 85 ans, les conducteurs électriques déposés sont jugés dans un état correct par les opérateurs.

De ce fait, RTE a lancé plusieurs projets ambitieux de R\&D ces dernières années, dans le but de mieux appréhender les phénomènes liés au vieillissement des conducteurs de son parc. L'objectif majeur de cette démarche d'envergure est de pouvoir distinguer les lignes aériennes (ou les portées ${ }^{1}$ les constituant) en fonction de leur vécu et des sollicitations externes leur ayant été appliquées. Les méthodologies de RTE pour remplir cet objectif reposent notamment, suivant les projets concernés, sur des campagnes de prélèvements in situ, des essais expérimentaux en laboratoire et des modélisations numériques. S'inscrivant dans cette démarche, le projet OLLA (acronyme de Overhead Lines Lifespan Assessment) vise à analyser les paramètres principaux influençant le vieillissement mécanique des conducteurs de lignes aériennes, par l'intermédiaire d'outils de simulation numérique. Plus précisément, ce projet est basé sur un volet de modélisations mécaniques et un volet d'analyses statistiques. L'originalité majeure de la démarche développée au sein de OLLA consiste en un dialogue entre les deux volets, permettant ainsi de combiner les avancées de chacun d'entre eux. Outre l'amélioration de la compréhension des phénomènes impliqués dans ce vieillissement mécanique des conducteurs, le but de OLLA est de fournir un interclassement des lignes permettant aux ingénieurs RTE d'obtenir un ordre de priorité pour le remplacement des conducteurs, tout en tenant compte des retours terrain.

\subsection{Présentation de l'objet d'étude du projet : les conducteurs de lignes aériennes}

Les conducteurs électriques de lignes aériennes sont constitués de plusieurs dizaines de brins torsadés sur plusieurs couches, les différents brins étant fabriqués dans un même matériau ou dans des matériaux différents. L'une des configurations les plus répandues se compose de brins circulaires en acier dans les couches centrales du conducteur et de brins circulaires en aluminium pour les couches externes. Les brins d'acier au cour procurent donc la résistance mécanique, et ceux en aluminium sur les couches externes ont pour rôle majeur la conduction du courant électrique. La fabrication de ces conducteurs, dits ACSR (Aluminium Conductors Steel Reinforced), est régie par des normes internationales [1]. Une représentation d'un tel type de conducteur est donnée sur la figure 1.

Concernant les caractéristiques géométriques des conducteurs ACSR utilisés sur le parc de RTE, les brins les constituant ont un diamètre compris entre 2 et 4,5 millimètres. De plus, ces brins sont le plus souvent disposés sur 4 à 6 couches, menant ainsi à un diamètre total du conducteur allant de 1,4 à 3,2 centimètres. Enfin, ces

\footnotetext{
${ }^{1}$ Une portée représente l'intervalle entre deux pylônes supports et est occupée par plusieurs conducteurs électriques (au moins un par phase). Une ligne aérienne est ainsi constituée de plusieurs dizaines de portées.
}

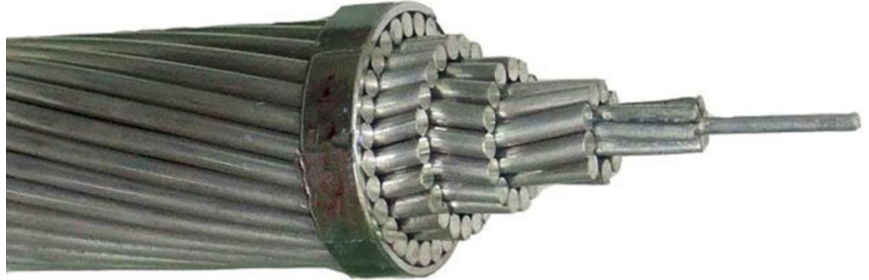

Fig. 1. Vue décomposée d'un conducteur électrique de ligne haute-tension à brins circulaires (source: Adto Group).

Fig 1. Cutaway view of a high-voltage line electrical conductor with circular strands (source: Adto Group).

brins sont enroulés successivement autour d'un brin central avec des pas d'enroulements qui varient suivant les couches concernées. Par application de la norme [1], ces pas d'enroulement sont classiquement compris dans un intervalle allant de 15 à 35 centimètres.

Ces conducteurs sont installés sur les lignes aériennes en suivant différentes étapes techniques. Tout d'abord, ils sont déroulés sur plusieurs portées successives et mis en position sur des poulies situées au niveau des pylônes (voir photo de gauche sur la Fig. 2). Durant cette opération, le conducteur est soumis à une tension mécanique, choisie de façon à éviter d'éventuelles proximités géométriques de la ligne avec l'environnement, notamment lorsque la température du conducteur augmente. Ensuite, une fois que l'équilibre statique de la ligne est établi, les poulies sont remplacées par des systèmes de fixation, appelées pinces (à droite sur la Fig. 2), de manière à garder la tension mécanique initiale. Une fois ces opérations réalisées sur l'ensemble des portées la composant, la ligne aérienne est mise sous tension électrique.

\subsection{Approche retenue pour le volet mécanique du projet}

Au regard de la description qui vient d'en être faite, il apparaît que les conducteurs de lignes aériennes sont des structures fortement hétérogènes, en tant qu'assemblages de plusieurs brins faits de différents matériaux. De plus, ces derniers sont déployés sur des portées dont la longueur est communément comprise entre 200 et 650 mètres. À titre d'exemple, la figure 3 représente la distribution de la longueur des portées pour un type de conducteur utilisé sur le parc de RTE. Enfin, comme cela va être évoqué en détails dans la suite de l'article, l'endommagement mis en lumière pour les conducteurs aériens se produit à une échelle très locale, impliquant le contact entre des brins.

Les considérations qui viennent d'être évoquées ont mené RTE à adopter, pour le volet mécanique de OLLA, une approche multi-échelle constituée de trois niveaux de modélisation numérique. Sans entrer dans les détails de ces modèles, la décomposition représentée sur la figure 4 a été retenue. Le modèle simplifié employé à l'échelle de la portée évalue le comportement du conducteur complet vis-à-vis des sollicitations complexes issues de l'environnement qui lui sont appliquées, en particulier celles provenant du vent. La modélisation d'un tronçon de conducteur permet de rendre compte de l'interaction entre tous les brins le 

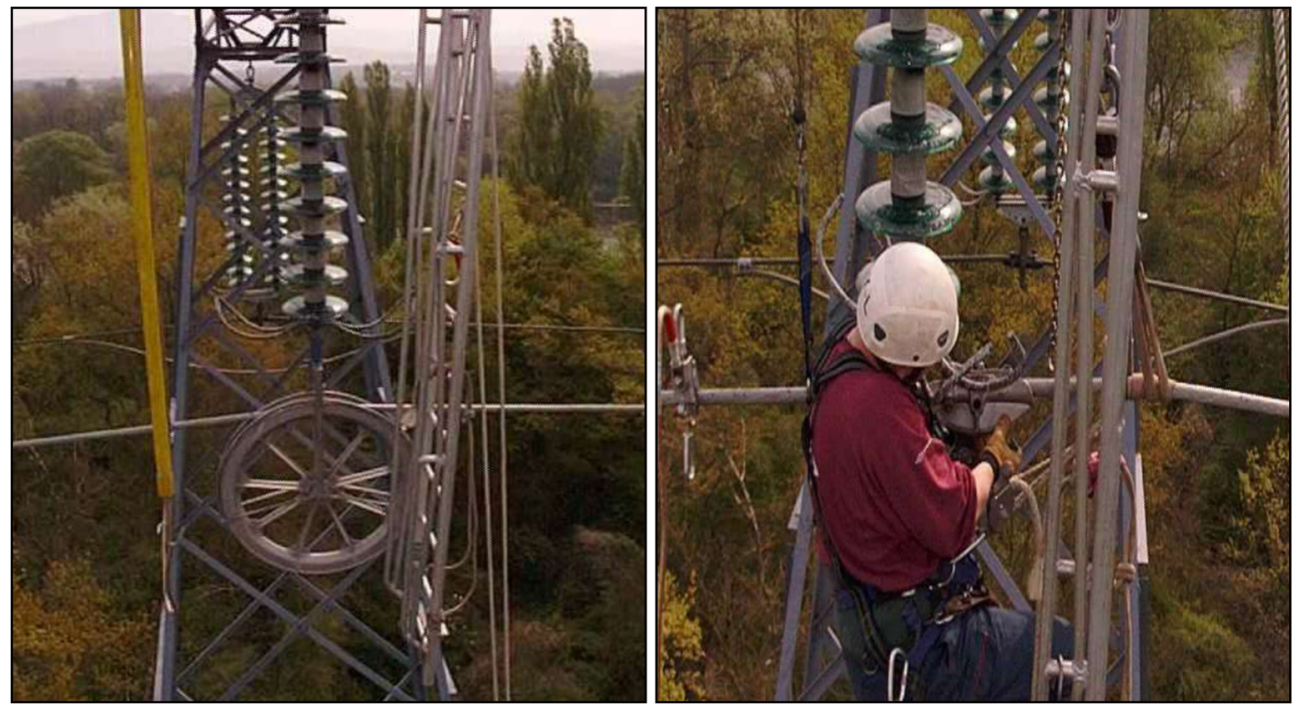

Fig. 2. Illustration de la mise en service des lignes aériennes : pose temporaire sur poulie (à gauche), puis mise sur pince (à droite).

Fig 2. Installation of overhead lines: initial laying on a pulley (left), then placing on clamp (right).

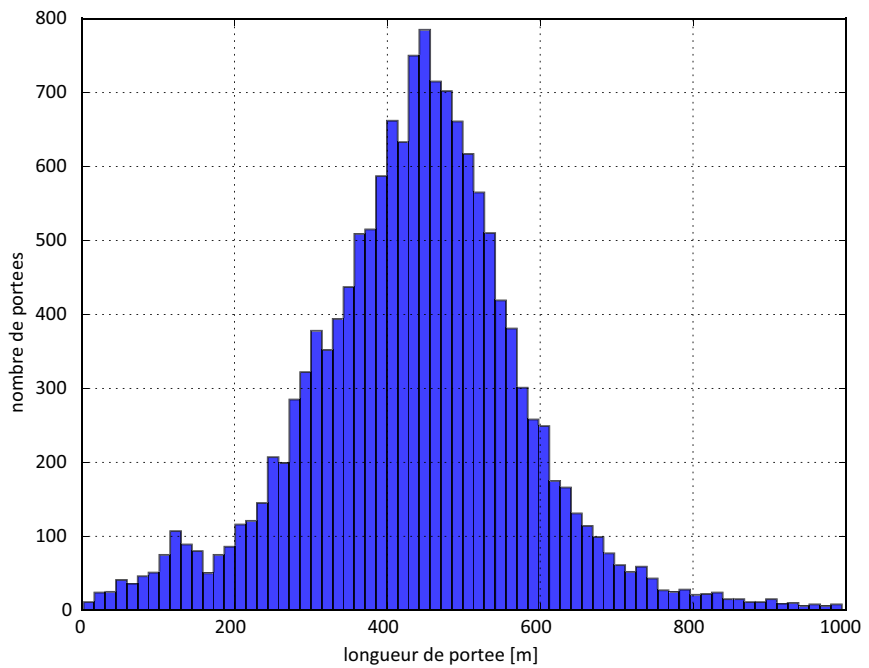

Fig. 3. Distribution de la longueur de portée des conducteurs ACSR de type Crocus 412 sur l'ensemble du territoire métropolitain français.

Fig 3. Distribution of Crocus 412 ACSR conductor span lengths throughout Metropolitan France.

constituant, ce qui conduit à détecter les zones de contact les plus critiques. Enfin, le modèle éléments finis à l'échelle d'un contact analyse de façon fine les champs de contrainte impliqués au niveau local par les chargements à l'échelle de la portée. Cette approche multi-échelle implique de façon évidente des transferts d'information entre les modèles numériques décrits.

Le présent article est ainsi dédié à l'échelle de modélisation la plus locale du volet mécanique du projet OLLA. Dans un premier temps, la section 2 décrit les systèmes de fixation en tant que zones critiques dans lesquelles des endommagements du conducteur sont particulièrement observés. Le phénomène de fretting-

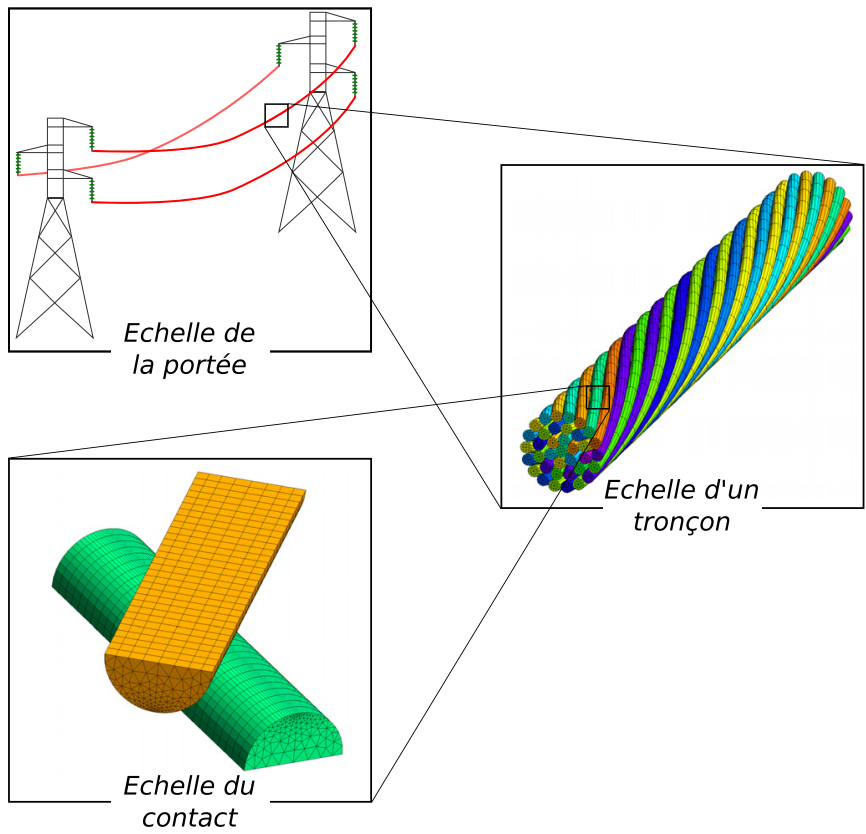

Fig. 4. Schématisation de l'approche multi-échelle du volet mécanique du projet OLLA.

Fig 4. Schematic of the multi-scale approach to the mechanical constituent of the OLLA project.

fatigue, identifié comme cause de ces endommagements, y est présenté. La section 3 aborde ensuite la méthodologie retenue pour évaluer la durée de vie des conducteurs vis-àvis de ce phénomène. Celle-ci fait intervenir des considérations sur les hypothèses à prendre en compte et les méthodes numériques à employer. À titre d'illustration, la section 4 fournit un premier exemple d'application de cette méthodologie sur un cas-test réaliste, dont les données d'entrée sont issues de la littérature. Cela permet en 


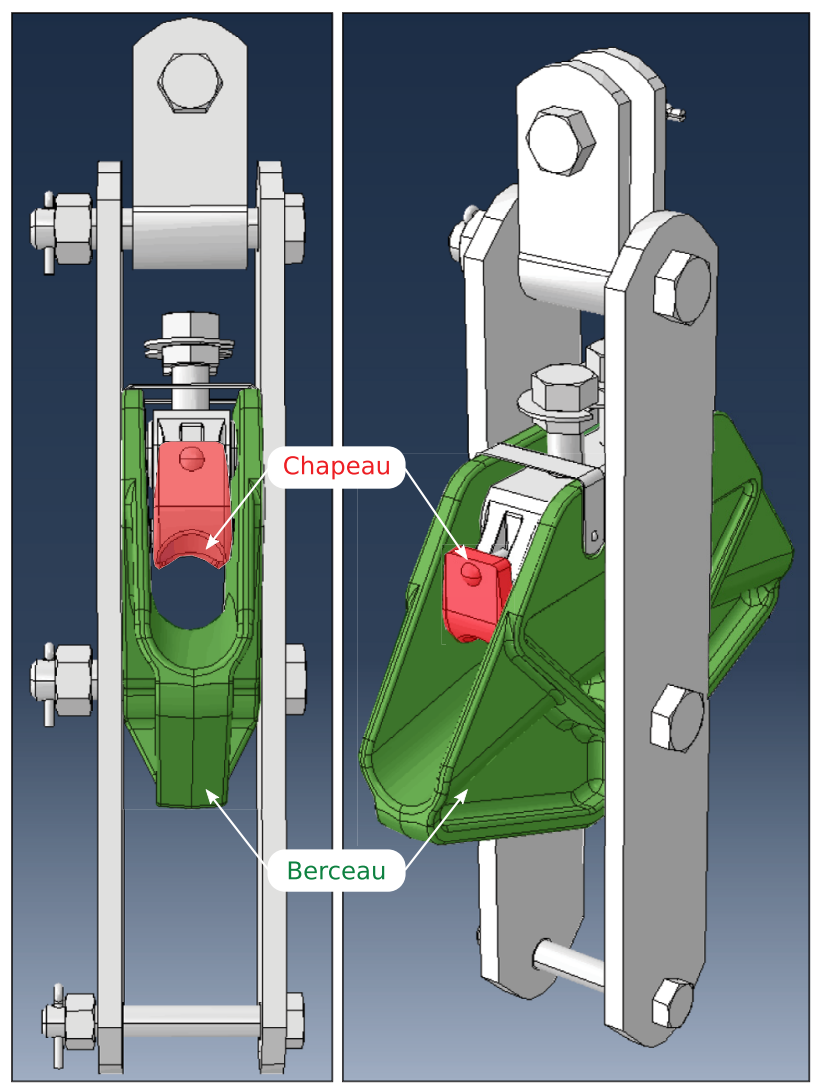

Fig. 5. Vues d'une pince de fixation, composée d'un berceau (en vert) et d'un chapeau (en rouge).

Fig 5. Views of a clamp, consisting of a clamp body (in green) and a keeper (in red).

section 5 de tracer de façon concrète les perspectives des travaux à venir à cette échelle et de leur intégration dans le projet OLLA.

\section{Description du lieu et du mécanisme d'endommagement étudiés}

\subsection{Présentation de l'interaction du système d'accroche avec le conducteur et des sollicitations associées}

Comme évoqué plus haut, les conducteurs de lignes aériennes sont fixés au niveau des pylônes supports. Il existe plusieurs types de système de fixation sur le parc de RTE. Cependant l'un de ceux les plus répandus est la pince de suspension. Les parties de la pince en contact avec le conducteur sont le berceau réceptacle de la partie inférieure du conducteur et le chapeau en partie supérieure. Des vues tridimensionnelles d'une telle pince sont représentées sur la figure 5. La majorité des pièces est fabriquée en alliage d'aluminium. Ces pinces sont dites à glissement contrôlé. Pour remplir cette fonction, les opérateurs effectuent un serrage entre le chapeau et le berceau de la pince, en appliquant des couples limites à des vis prévues à cet effet (au niveau du chapeau sur la Fig. 5).
Concernant les sollicitations appliquées au conducteur, en plus de la pression de serrage exercée par la pince, on peut en premier lieu recenser le poids propre $^{2}$ de la ligne, impliquant une flèche de quelques mètres en milieu de portée, et la tension mécanique qui fluctue autour de la valeur initiale à la pose, notamment en fonction de la température du conducteur. Bien que l'environnement de la ligne aérienne puisse engendrer divers types de chargement, il a été choisi lors du projet OLLA de focaliser les analyses mécaniques sur l'action des vents faibles sur la portée. En effet, même pour de petites vitesses de vent (inférieures à $7 \mathrm{~m} . \mathrm{s}^{-1}$ ), de nombreuses études ont prouvé l'existence de vibrations de faible amplitude avec des fréquences relativement élevées (jusqu'à $50 \mathrm{~Hz}$ ). Parmi ces recherches expérimentales, des études ont été effectuées par l'intermédiaire de mesures in situ [2] et d'essais en laboratoire [3]. Ces analyses expérimentales, ainsi que les retours d'expérience des opérateurs sur le terrain, ont conduit RTE à considérer comme prioritaire l'étude de ce phénomène vibratoire, nommé "vibrations éoliennes" (aeolian vibration en anglais) par opposition au phénomène de galop.

\subsection{Focus sur le mécanisme d'endommagement considéré}

Afin de comprendre l'assertion qui vient d'être faite, il est utile de souligner que les vibrations engendrées dans le conducteur par les vents faibles vont se produire pendant des millions de cycles au cours de la vie de la ligne aérienne. Cela se comprend d'autant mieux lorsqu'on précise que le vent moyen mesuré en France métropolitaine possède une vitesse médiane comprise entre $1 \mathrm{~m} . \mathrm{s}^{-1}$ et $5 \mathrm{~m} . \mathrm{s}^{-1}$, suivant les stations météo retenues sur le territoire. Ainsi, le conducteur est sollicité par des millions de cycles d'oscillation, de faible amplitude, autour de la position d'équilibre liée à son poids propre et à la tension mécanique appliquée.

Comme on peut l'imaginer, ces vibrations ont notamment des conséquences au niveau des pinces de fixation, puisqu'elles sont un lieu de concentration des contraintes pour le conducteur. Ainsi, lors de la visite régulière des lignes aériennes, les opérateurs observent parfois un ou plusieurs brins coupés sur le conducteur à proximité de la pince de fixation. La figure 6 montre ainsi un exemple de conducteur endommagé, pour lequel on constate des brins coupés qui se sont détoronnés, ce qui permet une détection visuelle des opérateurs depuis le sol. Bien que non-critiques pour la tenue mécanique lorsque leur nombre est limité, ces brins coupés nécessitent des opérations de réparation de la part de RTE. À propos des brins coupés, on souligne ici que la détection visuelle n'est possible que pour la couche externe de brins. Il est, en l'état actuel, impossible pour RTE de détecter la présence de brins coupés dans les couches internes d'un conducteur.

\footnotetext{
${ }^{2}$ La masse linéique des conducteurs ACSR utilisés classiquement par RTE est comprise entre $0,4 \mathrm{~kg} \cdot \mathrm{m}^{-1}$ et $2,3 \mathrm{~kg} \cdot \mathrm{m}^{-1}$.
} 


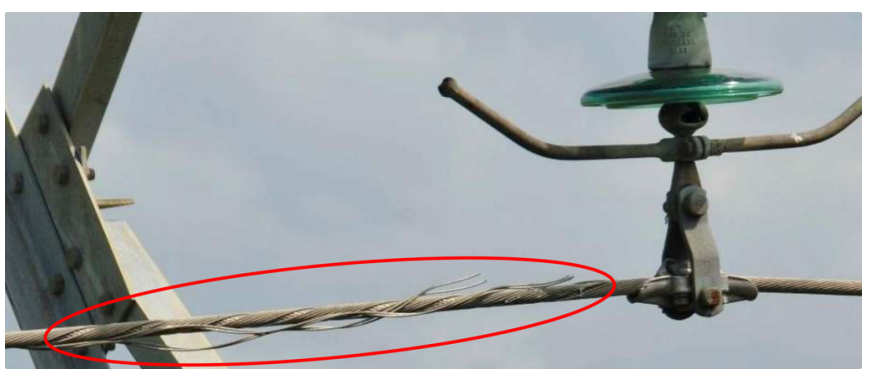

Fig. 6. Exemple d'observation de brins coupés sur un conducteur électrique de ligne aérienne.

Fig 6. Example of broken strands observed on an overhead line electrical conductor.

Parmi les investigations menées pour mieux appréhender le phénomène responsable de ces brins coupés, RTE a en particulier procédé à des prélèvements in situ de pinces ayant été en service pendant des décennies afin d'effectuer des analyses qualitatives. Comme le montrent les photos d'un berceau et d'un chapeau d'une de ces pinces sur la figure 7 , de fortes indentations sont observées en partie centrale de la pince. Celles-ci ont tendance à s'estomper pour faire place à une zone plus lisse, qui contient notamment le dernier point de contact (DPC) entre la pince et le conducteur.

En plus de ces observations, des recherches menées dans d'autres pays concluent au fait qu'une part des ruptures de brins d'aluminium sont imputables au phénomène de fretting-fatigue. Ce mécanisme d'endommagement peut être vu comme correspondant à une fatigue de contact par petits débattements $[4,5]$ et se rencontre dans divers assemblages industriels. Dans le cas spécifique des conducteurs électriques de lignes aériennes, les auteurs ont mis en évidence ce phénomène lors d'essais expérimentaux en laboratoire, en soulignant le fait que celui-ci est particulièrement prononcé au droit du DPC [6-8]. On peut au passage ajouter que ce type d'endommagement a été également observé in situ à proximité d'autres matériels se trouvant cette fois dans la partie courante de la portée [9]. En raison de ce constat, le fretting-fatigue est donc considéré dans le projet OLLA comme le phénomène d'endommagement à étudier pour évaluer le vieillissement mécanique des conducteurs de lignes aériennes.

\section{Définition de la méthodologie d'évaluation de la durée de vie de l'assemblage}

\subsection{Description des hypothèses employées pour cette évaluation}

À l'échelle du contact de l'approche multi-échelle de OLLA (voir Fig. 4), on cherche donc à évaluer les comportements locaux dans un conducteur vis-à-vis de sollicitations cycliques, issues du chargement du vent. Ces comportements locaux sont à étudier au niveau de certains contacts brin / brin et de l'interaction entre la pince et les brins de la couche externe du conducteur. Le but final est d'établir un classement relatif de l'état mécanique des conducteurs sur

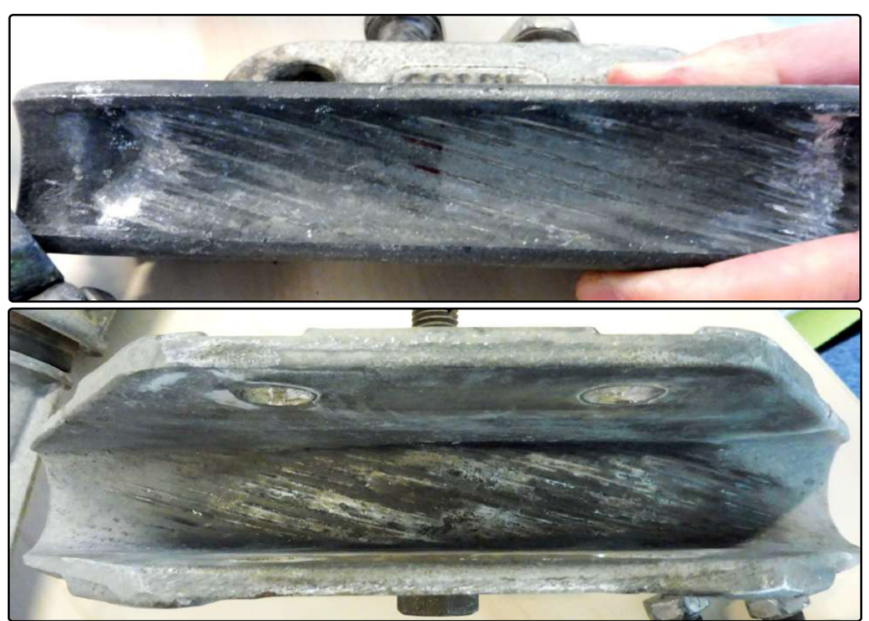

Fig. 7. Photographie de l'état de la surface d'un chapeau (en haut) et d'un berceau (en bas) d'une pince prélevée in situ à l'issue de plusieurs années de service.

Fig 7. Photograph of the surface condition of a keeper (top) and a clamp body (bottom) taken in situ after many years of service.

les différentes lignes du parc grâce à une approche comparative de leur durée de vie. Afin de pouvoir prendre en compte les configurations et les champs de contrainte complexes pouvant être rencontrés lors de la modélisation d'un tel problème, il a été choisi de se baser sur un modèle éléments finis du contact à analyser.

Pour évaluer la durée du vie au niveau du contact, on s'appuie tout d'abord sur le fait que le phénomène de fretting-fatigue apparaît pour un grand nombre de cycles de chargement. De ce fait, la prédiction de la durée de vie, qui sera exprimée en nombre de cycles, repose sur l'utilisation d'un critère d'amorçage de fissures. En effet, dans le cas de ce phénomène d'endommagement à grand nombre de cycles, seule la phase d'amorçage des fissures a été considérée, en faisant l'hypothèse d'un nombre de cycles de propagation non significatif de ces fissures. Cela est d'autant plus vrai que les brins des conducteurs ont de petits diamètres (inférieurs à $5 \mathrm{~mm}$ ). De plus, le fait d'effectuer cette hypothèse présente l'avantage de garder un aspect conservatif à la prédiction de la durée de vie.

Concernant les calculs, on cherche à déterminer un état stabilisé adapté au niveau du contact entre les surfaces considérées. Cet état est défini par le fait que, après avoir subi initialement des déformations plastiques dans une phase dite d'adaptation, l'ensemble des points des solides en contact se remet à suivre un trajet élastique [10]. C'est sur les champs de contrainte cycliques obtenus lors de cet état adapté que l'on cherche à évaluer le risque d'amorçage de fissures aux points les plus critiques. Au passage, on souligne qu'une telle approche permet donc de prendre en compte le changement de géométrie des surfaces en contact, induit par des contraintes dépassant les limites d'élasticité des matériaux. Dans le cas d'un conducteur en interaction avec une pince, une déformation plastique des brins est particulièrement possible lors de la phase de serrage de celle-ci. 


\subsection{Aspects techniques de la méthodologie employée}

Applicable à d'autres problèmes de mécanique, cette méthodologie d'évaluation de la durée de vie peut ainsi être résumée par les étapes suivantes dans le cas des conducteurs de lignes aériennes:

- application des chargements statiques (i.e. tension mécanique, poids propre, serrage de la pince);

- application de quelques cycles de chargement alterné d'amplitudes sévères, correspondant à l'interaction de la portée avec le vent;

- vérification de l'existence d'un état stabilisé adapté après ces cycles;

- si cet état est atteint en tout point, utilisation d'un critère d'amorçage de fissures sur les champs de contrainte recueillis au niveau des points les plus sollicités au cours du chargement cyclique.

Des précisions sur les choix effectués dans le cadre du projet OLLA sur cette méthodologie originale doivent être données. Tout d'abord, le logiciel éléments finis Abaqus [11] a été retenu pour modéliser le contact concerné. Pour le calcul de l'état stabilisé adapté, il existe plusieurs méthodes telles que la méthode stationnaire incrémentale [12], la méthode à grand incrément de temps [13] ou encore la méthode cyclique directe [14]. Les résultats qui seront présentés dans la suite de cet article sont obtenus avec la méthode incrémentale stationnaire, classiquement utilisée dans les simulations numériques de ce type. Il faut noter à ce stade que l'état stabilisé adapté n'est pas forcément observé suivant le nombre de cycles de chargement alterné appliqués. Le choix étant subjectif, on peut l'augmenter dans le cas où tous les points des solides en contact n'auraient pas été atteint cet état. Pour des raisons de coûts de calcul, on cherchera toutefois à minimiser le nombre de cycles de chargement alterné à appliquer au modèle. Une observation des champs de contrainte des points les plus sollicités est donc nécessaire pour déterminer si cet état stabilisé adapté est atteint.

Comme cela vient d'être évoqué, l'évaluation de la durée de vie du contact se fait en appliquant un critère d'amorçage de fissures à partir du champ de contrainte obtenu. Or, si les mécanismes d'amorçage des fissures en fretting-fatigue sont bien appréhendés de façon qualitative, l'estimation quantitative de la durée de vie reste complexe, compte tenu de la difficulté de connaître les champs de contrainte et de déformation, présents localement. Les démarches actuelles consistent à transposer les approches classiques de fatigue au problème de fretting-fatigue. Ainsi, le risque d'amorçage est évalué en estimant par le calcul l'état de contrainte imposé au niveau du contact, puis en comparant cet état de contrainte à un seuil d'amorçage défini par le critère de fatigue retenu [15]. Le critère d'amorçage de fissures à utiliser doit ainsi permettre de prendre en compte l'aspect multi-axial des champs de contrainte attendus au niveau microscopique du contact.

Dans le présent article, il a donc été décidé d'effectuer les analyses en se basant sur le critère de Dang Van $[16,17]$. Son utilisation combinée avec des simulations numériques a notamment été appliquée avec succès dans la littérature [18].

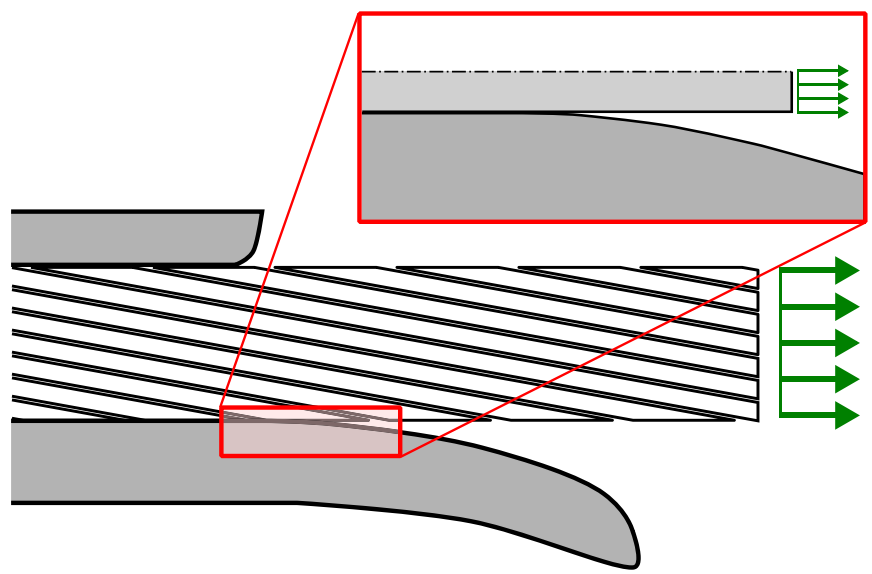

Fig. 8. Schématisation du chargement du contact brin/pince en phase 1 - mise en tension du conducteur.

Fig 8. Schematic of the strand/clamp contact loading in phase 1-tensile loading of the conductor.

De tels calculs nécessitent en particulier la détermination sur la solution numérique de deux quantités remarquables : la contrainte de cisaillement microscopique alternée $^{3} \tau_{\mathrm{a}}$ et la contrainte hydrostatique $p$. Dans le cadre du projet OLLA, les données d'entrée du modèle proviennent essentiellement de la descente d'échelles impliquant les différentes modélisations des conducteurs.

\section{Exemple d'application de la méthodologie}

\subsection{Présentation des données d'entrée de l'application}

À titre d'illustration de la méthodologie qui vient d'être exposée, ayant pour objectif d'évaluer la durée de vie d'un contact représentatif d'un conducteur de ligne aérienne, on propose ici d'utiliser des données expérimentales issues de la littérature. Pour cela, on s'appuie sur des travaux de simulation numérique visant à reproduire le comportement du contact brin / pince lors d'un essai de mise en vibration d'un conducteur [19]. Les données d'entrée du modèle éléments finis proposé par les auteurs avaient été évaluées de façon expérimentale lors d'un essai sur banc de vibration de longueur réduite $(7 \mathrm{~m})$. Ces mesures consistaient à relever, par l'intermédiaire de jauges apposées sur un brin externe du conducteur, les déformations de ce dernier à proximité du DPC, notamment au cours d'une excitation verticale sinusoïdale [20-22].

De façon plus précise, Lévesque et al. [19] ont cherché à simuler les phases de service d'un conducteur, décrites en section 1.2. Ainsi, les auteurs ont tout d'abord modélisé la phase de mise en tension mécanique en imposant localement au brin une traction longitudinale (voir Fig. 8). La contrainte associée est évaluée grâce à la mesure de la déformation du brin pour cette phase et par application de la loi de Hooke ${ }^{4}$.

\footnotetext{
${ }^{3}$ Les détails permettant de calculer $\tau_{\mathrm{a}}$ sont donnés dans les articles de référence [16,17].

${ }^{4}$ Cette remarque implique que, à l'échelle du brin, Lévesque et al. [19] ont estimé que son comportement restait élastique.
} 


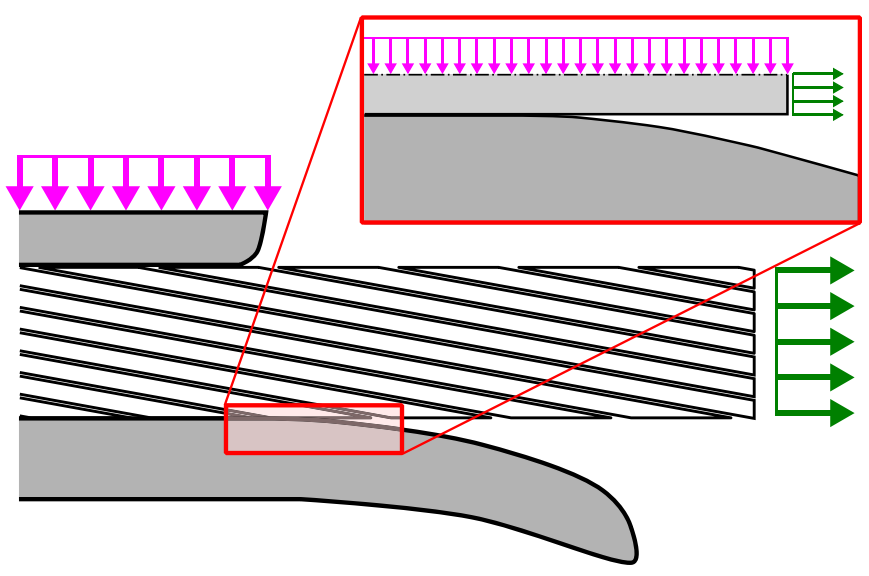

Fig. 9. Schématisation du chargement du contact brin/pince en phase 2 -serrage de la pince.

Fig 9. Schematic of the strand/clamp contact loading in phase 2-tightening of the clamp.

La deuxième phase simulée correspond au serrage de la pince, le conducteur étant toujours soumis à la même tension mécanique. Cela implique pour les auteurs un déplacement vertical du chapeau qui se répercute au niveau du brin (voir Fig. 9). Pour évaluer ce rapprochement du brin vers la pince, Lévesque et al. ont procédé à des simulations numériques de façon à obtenir une bonne adéquation entre l'aire de contact mesurée lors de l'essai et celle obtenue par calcul, en considérant un comportement élastoplastique des composants en contact.

Pour finir, les simulations portent sur la mise en vibration du conducteur soumis aux deux sollicitations précédemment exposées. Cette phase comprend des instants pour lesquels l'amplitude de vibration est minimale (vers le bas) et d'autres où elle est maximale (vers le haut). Pour la suite du document, on note ces phases respectivement $3^{-}$et $3^{+}$. Conceptuellement, cela revient à considérer que le brin se situant au DPC avec la pince subit une traction ondulée (voir Fig. 10), dont l'effort associé est également obtenu par des jauges de déformation et par application de la loi de Hooke. L'historique de cet effort est bien sûr relié à l'amplitude de vibration du conducteur. Comme pour l'ensemble des phases, un lecteur intéressé trouvera tous les détails des valeurs numériques mesurées dans l'article de référence [19].

Les expériences ont été menées sur un conducteur ACSR de type Bersfort. Ce dernier est employé notamment sur le réseau d'électricité haute-tension au Canada. Il est composé de deux couches de brins d'acier (incluant le cœur) et de trois couches de brins d'aluminium. Les caractéristiques géométriques de ces couches sont données dans le tableau 1. Celles-ci procurent au conducteur Bersfort une section de $746,9 \mathrm{~mm}^{2}$ et un diamètre extérieur de $35.6 \mathrm{~mm}$

La zone modélisée correspond à un morceau de brin en aluminium de la couche externe (numérotée 4) en contact avec une partie de la pince, également en aluminium. Lévesque et al. [19] spécifient que les brins de cette couche sont constitués d'un aluminium pur de nuance 1350-H19, la

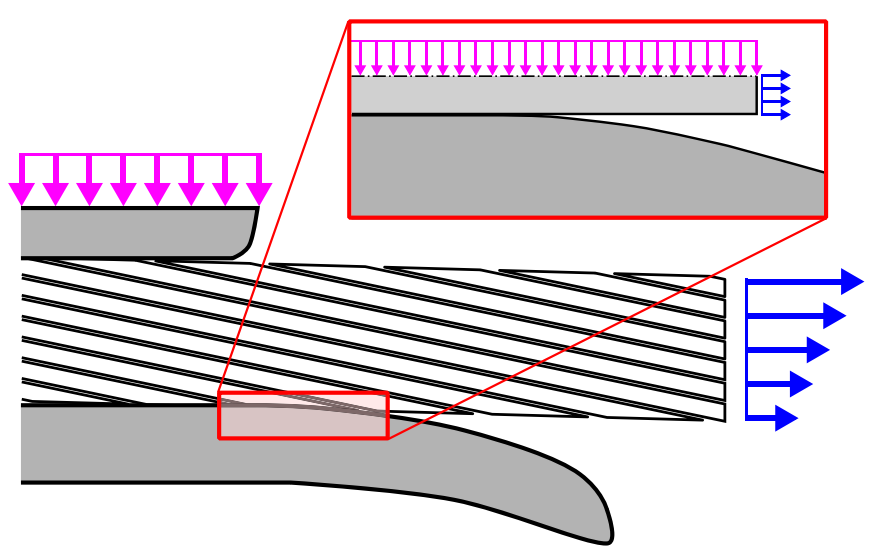

(a) phase $3^{-}$- amplitude minimale

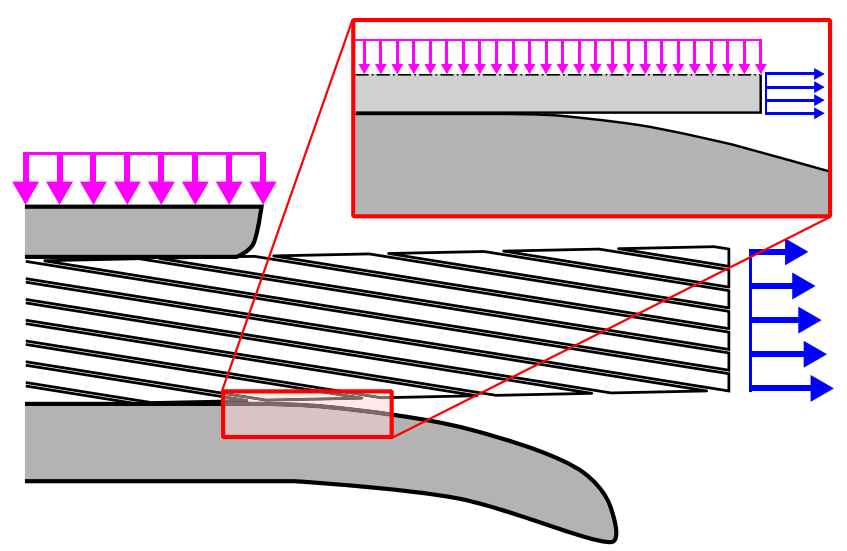

(b) phase $3^{+}$- amplitude maximale

Fig. 10. Schématisation du chargement du contact brin/pince en phase 3 -vibration du conducteur.

Fig 10. Schematic of the strand/clamp contact loading in phase 3-vibration of the conductor.

Tableau 1. Caractéristiques géométriques du conducteur ASCR de type Bersfort.

Table 1. Geometric characteristics of the Bersfort ACSR conductor.

\begin{tabular}{lcll}
\hline $\begin{array}{l}\text { Numéro de } \\
\text { la couche }\end{array}$ & $\begin{array}{l}\text { Nombre } \\
\text { de brins }\end{array}$ & $\begin{array}{l}\text { Diamètre des } \\
\text { brins }[\mathrm{mm}]\end{array}$ & $\begin{array}{l}\text { Nature des } \\
\text { brins }\end{array}$ \\
\hline Coeur & 1 & 3,32 & Acier \\
1 & 6 & 3,32 & Acier \\
2 & 10 & 4,27 & Aluminium \\
3 & 16 & 4,27 & Aluminium \\
4 & 22 & 4,27 & Aluminium \\
\hline
\end{tabular}

pince étant supposée être fabriquée dans le même matériau. Pour cet aluminium, les auteurs donnent les valeurs numériques estimées pour le module d'Young E, le coefficient de Poisson $v$ et la limite d'élasticité $S_{\mathrm{Y}}$, i.e:

$$
E=69 \mathrm{GPa}, v=0,33 \text { et } S_{\mathrm{Y}}=167 \mathrm{MPa} .
$$


Classiquement, le coefficient de frottement $\mu$ entre deux brins de conducteur constitués d'aluminium est considéré dans la littérature comme étant compris entre 0,3 et 0,7. Pour la suite de la modélisation, on choisit arbitrairement de prendre $\mu=0,3$.

Dans la méthodologie proposée à la section précédente, il est nécessaire de pouvoir construire le critère d'amorçage de fissures associé au matériau concerné. En l'occurrence, on souhaite pouvoir évaluer la droite de Dang $\operatorname{Van}^{5}$ pour un aluminium 1350-H19. Or pour ce faire, il est nécessaire de connaître les performances de ce matériau vis-à-vis de la fatigue, caractéristiques mécaniques non abordées par Lévesque et al. [19]. Étant donné le statut de preuve de concept de la présente application, l'évaluation de la droite de Dang Van est effectuée ici sans passer par des essais de fatigue dédiés. En se basant sur un catalogue de matériaux en ligne ${ }^{6}$, on considère donc que la limite d'endurance $\sigma_{\mathrm{D}}$ d'un aluminium 1350-H19 vaut $50 \mathrm{MPa}$, correspondant à la résistance en fatigue à $10^{7}$ cycles. Par la même source, la contrainte limite ultime $R_{m}$ est évaluée à $180 \mathrm{MPa}$. En appliquant une approche empirique [23], basée notamment sur l'utilisation de la droite de Goodman dans le diagramme de Haigh, on obtient les valeurs estimées suivantes des paramètres du critère de Dang Van pour l'aluminium concerné :

$$
a=-0,58 \text { et } b=34,62 \mathrm{MPa} .
$$

\subsection{Description du modèle éléments finis}

À l'instar des caractéristiques de l'essai simulé, le modèle éléments finis employé dans Abaqus reproduit, du point de vue de la géométrie, celui de l'article de référence [19]. Ainsi, en s'appuyant sur les symétries géométriques et du chargement imposé, il est possible de réduire la modélisation à un quart de tronçon de brin, en contact avec une partie de pince, à proximité du DPC. La géométrie retenue est représentée sur la figure 11, avec les conditions aux limites imposées sur les différentes surfaces de celle-ci. Au passage, on souligne que, en accord avec la description faite à la section précédente, le déplacement vertical imposé $u^{\text {imp }}$ n'est actif qu'à partir de la phase 2 (serrage de la pince) et la contrainte imposée sur l'une des extrémités $\mathrm{du}$ brin $\sigma^{\mathrm{imp}}$ est constante pour les phases 1 et 2 , puis fluctue autour de cette valeur moyenne dans les différents cycles de la phase 3 .

Tirant profit des analyses réalisées par Lévesque et al., le maillage retenu est directement raffiné dans les zones de plus grand intérêt. En l'occurrence, il s'agit ici du lieu de contact entre le brin et la partie de pince. Cela permet de capter au mieux les concentrations de contrainte qui s'y présentent. Des éléments finis tétraédriques avec une interpolation quadratique du déplacement sont utilisés.

\footnotetext{
${ }^{5}$ Pour rappel, la droite de Dang Van s'écrit sous la forme $\tau_{\mathrm{a}}+a p=b$.

${ }^{6}$ https://www.makeitfrom.com/material-properties/1350-H19Aluminum.
}

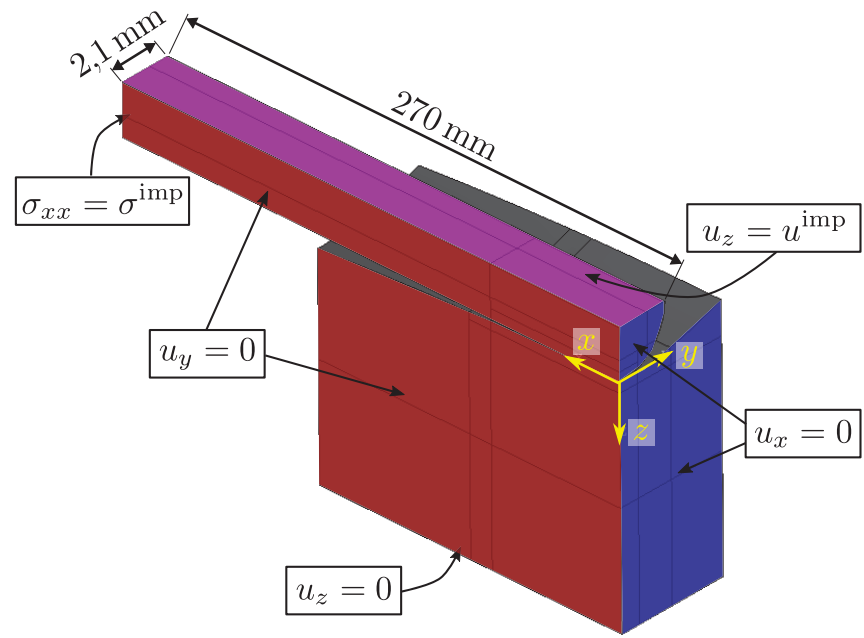

Fig. 11. Modèle éléments finis d'un brin en contact avec la pince (d'après Lévesque et al. [19]).

Fig 11. Finite element model of a strand in contact with the clamp (following Lévesque et al.).

Concernant la variation de $\sigma^{\text {imp }}$ lors de la phase 3 , il a été décidé ici de procéder à 12 cycles de chargement. Le brin est donc soumis alternativement 12 fois à la contrainte maximale correspondant à la flexion d'amplitude maximale, puis à la contrainte minimale issue de la flexion d'amplitude minimale (voir Fig. 10). Comme cela sera souligné dans la section d'analyse des résultats, ce nombre de cycles permet d'obtenir un état stabilisé adapté. Pour la modélisation dans Abaqus, on rappelle que les deux solides en contact sont constitués d'un matériau suivant une loi de type plasticité parfaite (sans écrouissage) et que l'approche numérique employée pour déterminer l'état adapté est une méthode stationnaire incrémentale.

\subsection{Analyse des résultats numériques obtenus}

La simulation a été lancée sur 12 cœurs d'un ordinateur PC (Intel Xeon E5-2697 v2, 2,7 GHz) pour ce modèle contenant 96427 nœuds (289281 degrés de liberté). La solution numérique pour les phases 1 et 2 ainsi que les 12 cycles de chargement de la phase 3 , correspondant au total à 482 pas de calcul, est obtenue en 2100 minutes, soit 33,5 heures.

Dans un premier temps, on vérifie bien que l'état stabilisé adapté est atteint pour le douzième cycle de chargement. Pour cela, le point le plus sollicité dans le brin est recherché en évaluant la contrainte de Von Mises obtenue sur ce solide pour tous les pas de chargement confondus. Ce dernier est localisé à proximité du dernier lieu de contact entre la pince et le brin (voir Fig. 12).

Sur la figure 13, on constate ainsi que le trajet de chargement, tracé ici dans le plan déformation/contrainte principales majeures noté $\left(\varepsilon_{I}, \sigma_{I}\right)$, au niveau du point le plus sollicité devient stable au sens où il se superpose d'un cycle à l'autre, à partir du cycle 5 . La vérification de l'obtention de l'état stabilisé adapté a également été menée en analysant les déformations plastiques au cours du calcul complet. Si ces dernières sont dues principalement aux 


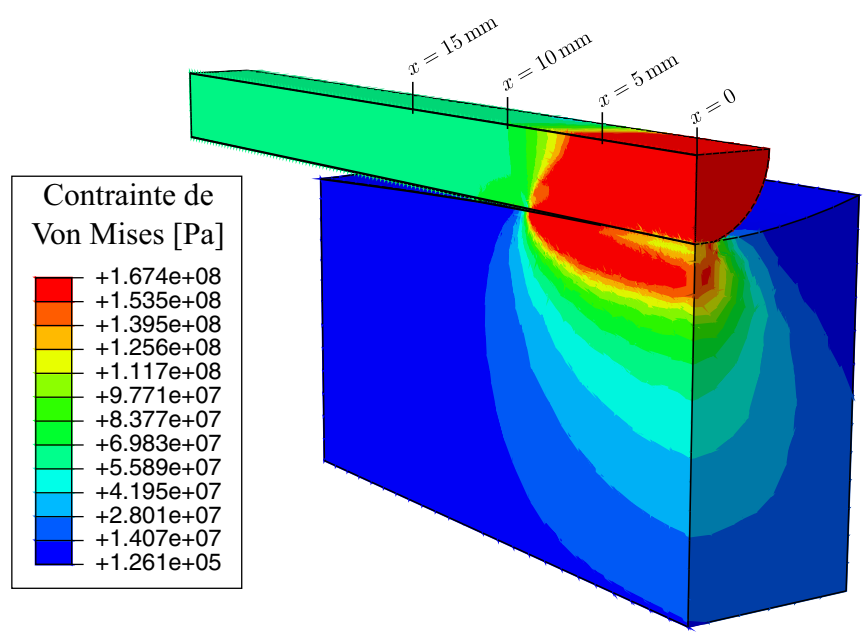

Fig. 12. Champ de contrainte de Von Mises pour le dernier pas du cycle 12 de la phase 3 .

Fig 12. Von Mises stress field for the last step of cycle 12 of the $3 r d$ phase.

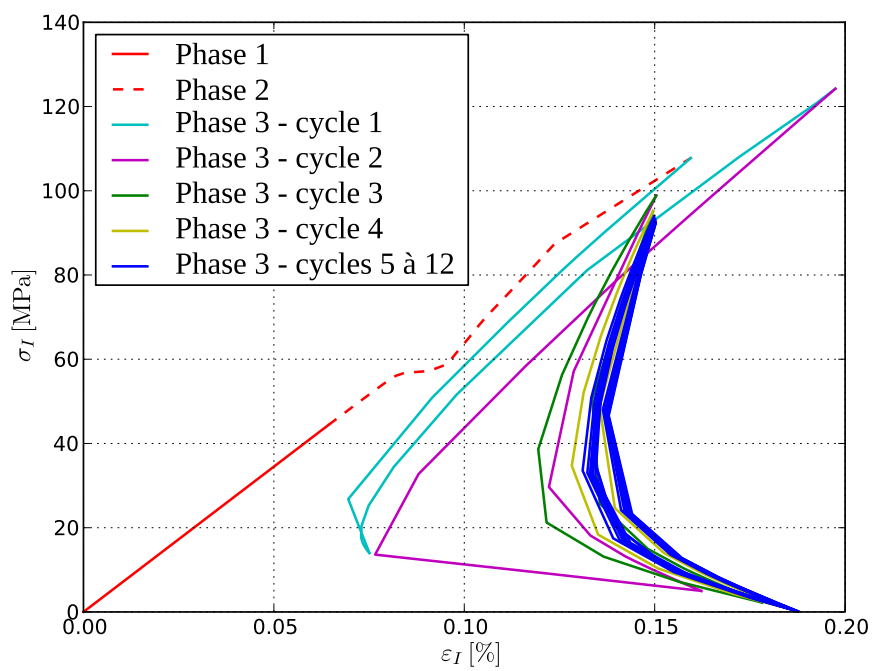

Fig. 13. Historique en contrainte/déformation principales majeures, sur la ligne de contact du brin avec la pince à $x=6,82 \mathrm{~mm}$, pour les différentes phases de chargement.

Fig 13. First principal components of stress/strain, on the strand-clamp contact line at $x=6.82 \mathrm{~mm}$, for the different loading phases.

deux premières phases de chargement, pour le dernier cycle de la phase 3, elles n'évoluent pas de plus de $2 \%$ au maximum entre le début et la fin du cycle pour le point le plus sollicité.

Une fois cette vérification préliminaire effectuée sur les champs solution, on cherche dans un premier temps à déterminer les points les plus critiques, vis-à-vis du phénomène de fretting-fatigue, sur la ligne de contact du brin avec la pince. Pour cela, on trace sur la figure 14 la pression hydrostatique $p$ et la contrainte de cisaillement $\tau_{\mathrm{a}}$ en fonction de l'abscisse des points sur cette ligne. Les représentations de ces quantités sont données à la fin de la

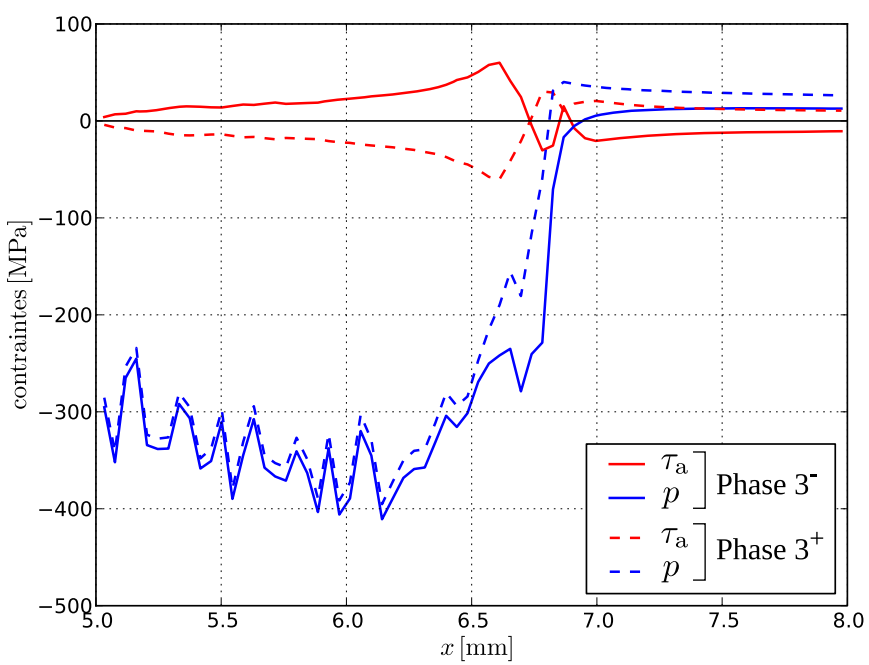

Fig. 14. Évolution de $p$ et de $\tau_{\mathrm{a}}$ sur une portion de la ligne de contact du brin avec la pince, pour la fin des phases $3^{+}$(lignes pointillées) et $3^{-}$(lignes pleines) lors du cycle 12 .

Fig 14. Evolution of $p$ and $\tau_{a}$ along the line of contact between strand and clamp, at the end of phases $3^{+}$(dotted lines) and $3^{-}$ (solid lines) during cycle 12.

phase $3^{+}$et à la fin de la phase $3^{-}$lors du douzième cycle de chargement. On constate alors que les amplitudes de $\tau_{\mathrm{a}}$ les plus grandes sont enregistrées pour une abscisse comprise entre $6,7 \mathrm{~mm}$ et $7 \mathrm{~mm}$. Toutefois, dans cette même zone, la pression hydrostatique connaît des variations importantes. La détermination du point le plus critique au regard du critère de Dang Van n'est donc pas évidente par la lecture de cette figure.

Afin d'effectuer cette détermination, un indicateur d'endommagement $\alpha$ est introduit, tel que :

$$
\alpha=\frac{b-\left(\left|\tau_{\mathrm{a}}(t)\right|+a p(t)\right)}{b} .
$$

Cette quantité est adimensionnelle et permet de situer le statut d'un point par rapport à la droite de Dang Van. Tel que souligné dans la littérature [14], pour tout point du solide, si $\alpha$ est négatif ou nul alors il existe un risque d'amorçage de fissures, en revanche si $\alpha$ est positif, alors ce risque est absent.

On représente ainsi sur la figure 15 l'évolution de $\alpha$ sur une portion de la ligne de contact pour les deux pas de chargement précédemment utilisés (pas ultimes des phases $3^{+}$et $3^{-}$du cycle 12 ). Il apparaît alors que le point pour lequel la valeur de cet indicateur est la plus faible correspond à $x=6,82 \mathrm{~mm}$. En ce point, $\alpha$ étant négatif, un risque d'amorçage de fissures est détecté. Ce constat est confirmé sur la figure 16 sur laquelle sont tracées, dans le plan $\left(p, \tau_{\mathrm{a}}\right)$, les trajets de chargement lors du cycle 12 de la phase 3 pour des nouds dont l'abscisse est comprise entre 6,7 et $7 \mathrm{~mm}$. Ainsi, pour $x=6,82 \mathrm{~mm}$, le chargement dépasse bien la droite de Dang Van (lignes rouges) pour des cisaillements positifs.

Enfin, on cherche à déterminer l'étendue de la zone critique vis-à-vis du phénomène de fretting-fatigue dans le brin, au droit du point d'abscisse $x=6,82 \mathrm{~mm}$ appartenant 


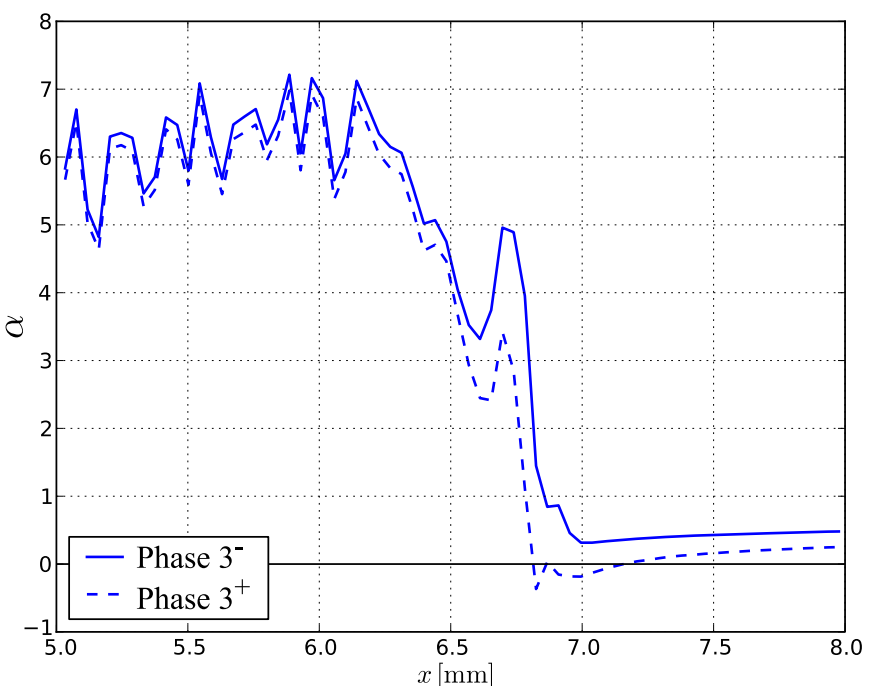

Fig. 15. Évolution de l'indicateur $\alpha$ sur une portion de la ligne de contact du brin avec la pince, pour la fin des phases $3^{+}$(ligne pointillée) et $3^{-}$(ligne pleine) lors du cycle 12 .

Fig 15. Evolution of the indicator $\alpha$ along the line of contact between strand and clamp, at the end of phases $3^{+}$(dashed line) and $3^{-}$(solid line) during cycle 12.

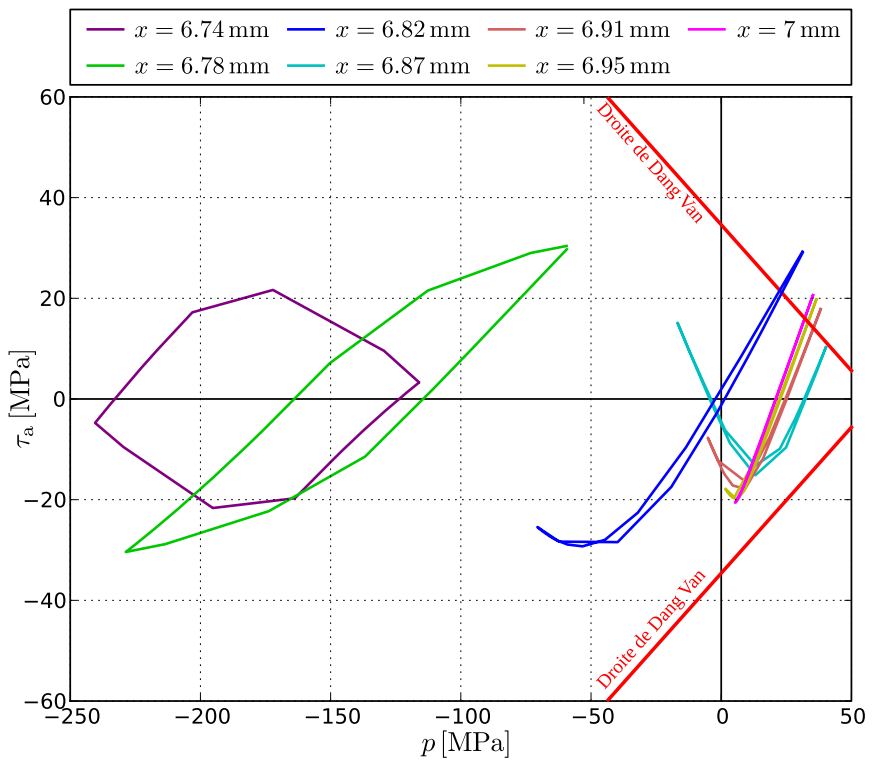

Fig. 16. Trajets de chargement dans le plan $p, \tau_{\text {a }}$ de plusieurs points de la ligne de contact du brin avec la pince, au cours du cycle 12 de la phase 3 .

Fig 16. Loading paths in the $p, \tau_{a}$ plane for several points along the contact between strand and clamp, during cycle 12 of the 3rd phase.

à la ligne de contact. Pour cela, on représente sur la figure 17 la cartographie dans la coupe concernée de l'indicateur d'endommagement $\alpha$, pour le douzième cycle de chargement, respectivement sous amplitude maximale et sous amplitude minimale. On s'aperçoit tout d'abord qu'aucun risque d'amorçage de fissures n'est détecté pour la fin de la phase $3^{-}$. En effet, quel que soit le point observé,

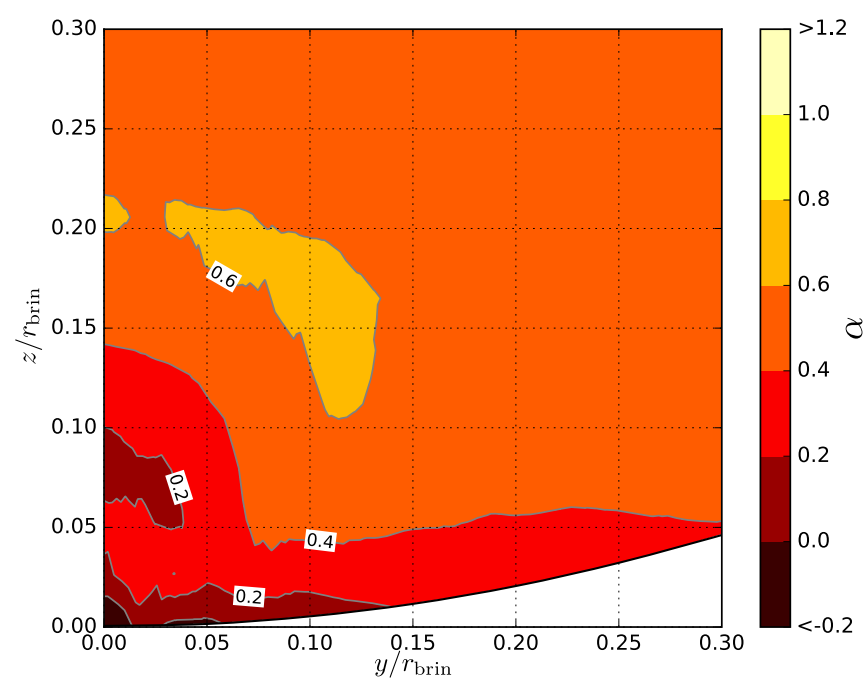

(a) fin de la phase $3^{+}$

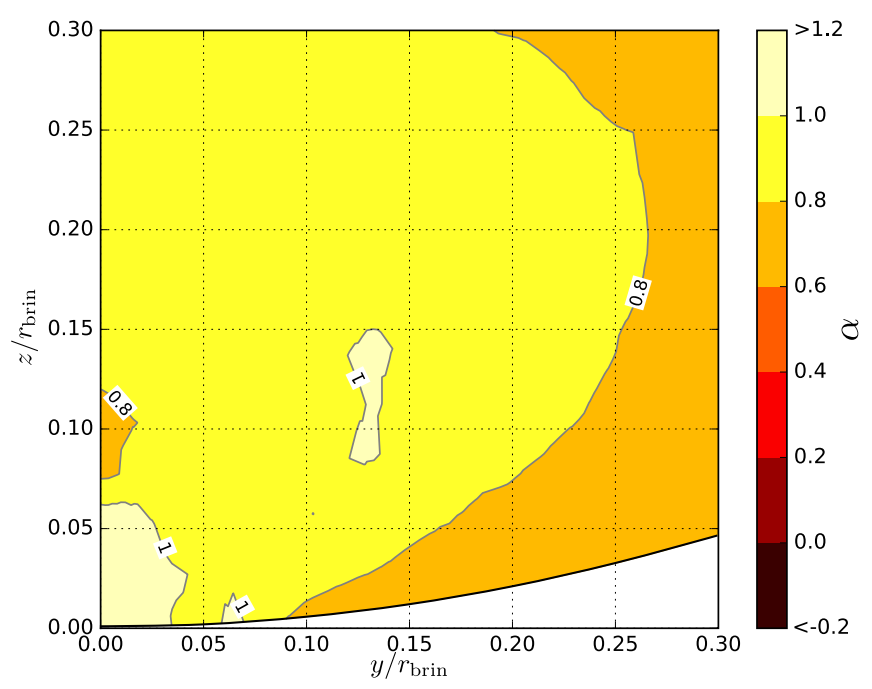

(b) fin de la phase $3^{-}$

Fig. 17. Cartographie de l'indicateur $\alpha$ dans la coupe $x=6,82 \mathrm{~mm}$, la fin des phases $3^{+}$et $3^{-}$lors du cycle 12 .

Fig 17. Mapping the $\alpha$ indicator in the $x=6.82 \mathrm{~mm}$ cross section, at the end of the $3^{+}$and $3^{-}$phases of Cycle 12 .

l'indicateur reste positif. Ensuite, on constate qu'à la fin de la phase $3^{+}$, une zone entourant le point appartenant à la ligne de contact avec la pince présente un risque de fissuration supérieur au reste de la section. On peut toutefois souligner qu'à la profondeur normalisée de $0,08 \times r_{\text {brin }}, \alpha$ prend des valeurs proches de zéro.

Plus que les résultats quantitatifs, il est important de souligner les méthodes d'analyse qui ont été employées ici. En effet, en associant l'étude des contraintes microscopiques à celle d'un indicateur basé sur le critère de Dang Van, la détermination de la zone la plus critique vis-à-vis de l'endommagement par fretting-fatigue a été effectuée. De telles analyses peuvent être menées sur d'autres types de problème de contact, à condition que l'état stabilisé adapté ait été atteint en tout point des solides étudiés. 


\section{Perspectives de développement et d'utilisation d'une telle approche}

Dans cet article, la démarche globale, liée au projet intitulé OLLA, visant à évaluer l'endommagement des conducteurs de lignes aériennes du parc de RTE a été présentée. Celle-ci repose sur une approche multi-échelle dont les différents modèles numériques impliqués ont été succinctement évoqués. Étant donné son rôle final dans l'évaluation de la durée de vie d'un conducteur, le modèle permettant de simuler le contact à l'échelle locale a été mis en avant. Plus que les résultats quantitatifs à tirer d'un tel modèle, la présente publication a eu pour objectif de décrire la méthodologie, originale dans le contexte, menant à une telle évaluation. Pour cela, les hypothèses principales, en particulier le choix du fretting-fatigue comme phénomène d'endommagement, ont été présentées ainsi que les simplifications de calcul associées.

Une application de cette méthodologie a été proposée en se basant sur des données d'entrée issues d'un essai expérimental de la littérature. Cette application correspond donc ici à une première preuve de faisabilité de la démarche numérique à l'échelle locale. Elle a notamment été l'occasion de fournir des indications techniques sur les analyses à mener à cette échelle de modélisation, en utilisant un logiciel de calcul éléments finis standard, i.e. Abaqus. Les étapes de calcul et de post-traitement constituant ces analyses ont été données et peuvent être appliquées à d'autres configurations de contact.

Sur le modèle numérique proposé en tant que tel, deux pistes de travail majeures restent à explorer prochainement. Tout d'abord, il s'agira d'évaluer l'influence du critère multi-axial d'amorçage de fissures sur la prédiction effectuée en termes de nombre de cycles de chargement supportables. Pour cela, on peut évoquer différents critères de la littérature, tels que Crossland [24], Smith-WatsonTopper [25] ou Ruiz [26] entre autres. De la même façon, on cherchera à utiliser différentes méthodes de résolution numérique visant à déterminer l'état stabilisé adapté au niveau du contact. Il s'agira d'évaluer l'influence de la méthode sur la solution obtenue, mais également sur le coût de calcul.

Dans son intégration au sein du projet OLLA, le même type de calcul devra être mené sur des contacts brin / brin ou brin / pince avec des données d'entrée issues de la chaîne de calculs multi-échelles présentée en section 1.3. Les questions de transfert d'informations entre les échelles devront être regardées avec attention. De plus, les performances du modèle éléments finis employé seront également un enjeu important. Au final, les modélisations mécaniques à différentes échelles pourront aboutir à un banc d'essai numérique permettant d'évaluer la durée de vie de plusieurs assemblages pince / conducteur. Ces durées de vie seront exprimées pour une amplitude de vibration donnée afin d'évaluer l'endommagement d'un assemblage en fonction de son historique de sollicitation. Ces informations seront cruciales pour RTE, mais les modélisations numériques n'éviteront pas la nécessité d'essais expérimentaux, et au contraire se nourriront de leurs résultats. En particulier, comme cela a pu être souligné, les performances en fatigue des matériaux constituant les brins du conducteur et la pince seront des données majeures intégrées dans la méthodologie présentée dans cet article.

Remerciements. Les auteurs tiennent à remercier Mr Minh Chien Nguyen pour ces travaux sur les modélisations éléments finis à l'échelle du contact. Celles-ci seront présentées avec plus de détails dans un article en préparation [27].

\section{References}

1. Norme Européenne, Conducteurs pour lignes aériennesConducteurs à brins circulaires, câblés en couches concentriques, NF EN 50182, 2001

2. P. Van Dyke, C. Hardy, M. St-Louis, J.-L. Gardes, Comparative field tests of various practices for the control of wind-induced conductor motion, IEEE Trans. Power Deliv. 12, 1029-1034 (1997)

3. P.-O. Dallaire, Étude des vibrations éoliennes instationnaires, Thèse de master, Université de Sherbrooke, Canada, 2008

4. D. Nowell, D.A. Hills, Mechanics of fretting fatigue tests, Int. J. Mech. Sci. 29, 355-365 (1987)

5. D.A. Hills, Mechanics of fretting fatigue, Wear 175, 107-113 (1994)

6. Z.R. Zhou, A. Cardou, S. Goudreau, M. Fiset, Fretting patterns in a conductor-clamp contact zone, Fatigue Fract. Eng. Mater. Struct. 17, 661-669 (1994)

7. B. Ouaki, S. Goudreau, A. Cardou, M. Fiset, Fretting fatigue analysis of aluminium conductor wires near the suspension clamp: metallurgical and fracture mechanics analysis, J. Strain Anal. Eng. Des. 38, 133-147 (2003)

8. C.R.F. Azevedo, A.M.D. Henriques, A.R. Pulino Filho, J.L. A. Ferreira, J.A. Araújo, Fretting fatigue in overhead conductors: Rig design and failure analysis of a Grosbeak aluminium cable steel reinforced conductor, Eng. Fail. Anal. 16, 136-151 (2009)

9. C.R.F. Azevedo, T. Cescon, Failure analysis of aluminum cable steel reinforced (ACSR) conductor of the transmission line crossing the Parana River, Eng. Fail. Anal. 9, 645-664 (2002)

10. S. Fouvry, P. Kapsa, L. Vincent, An elastic-plastic shakedown analysis of fretting wear, Wear 247, 41-54 (2001)

11. ABAQUS/Standard User's Manual, Version 6.14, Simulia, 2014

12. K. Dang Van, M.H. Maitournam, Elasto-plastic calculations of the mechanical state in reciprocating moving contacts: Application to fretting fatigue, in: Fretting Fatigue, Mechanical Engineering Publications, London, 1994, pp. 161-168

13. P. Ladevèze, La méthode à grand incrément de temps pour l'analyse de structures à comportement non linéaire décrit par variables internes, Comptes-rendus l'Acad. Sci. Série II 309, 1095-1099 (1989)

14. N. Maouche, Modélisation des phénomènes d'endommagements dus aux contacts à faible amplitude de débattement, Thèse de doctorat, École Nationale des Ponts et Chaussées, France, 1997

15. E. Pierres, Simulation tridimensionnelle multi-échelle de la propagation de fissures expérimentales sous chargement de fretting fatigue par la méthode des éléments finis étendus, Thèse de doctorat, INSA Lyon, France, 2010 
16. K. Dang Van, B. Griveau, O. Message, On a new multiaxial fatigue limit criterion: Theory and application, in: Biaxial and Multiaxial Fatigue, Mechanical Engineering Publications, London, 1989, pp. 479-496

17. K. Dang Van, Macro-micro approach in high-cycle multiaxial fatigue, in: Advances in Multiaxial Fatigue, ASTM International, Philadelphie, 1993

18. C. Petiot, L. Vincent, K. Dang Van, N. Maouche, J. Foulquier, B. Journet, An analysis of fretting-fatigue failure combined with numerical calculations to predict crack nucleation, Wear 181, 101-111 (1995)

19. F. Lévesque, S. Goudreau, L. Cloutier, A. Cardou, Finite element model of the contact between a vibrating conductor and a suspension clamp, Tribol. Int. 44, 1014-1023 (2011)

20. F. Lévesque, S. Goudreau, A. Cardou, L. Cloutier, Strain measurements on ACSR conductors during during fatigue tests I-Experimental method and data, IEEE Trans. Power Deliv. 25, 2825-2834 (2010)

21. S. Goudreau, F. Lévesque, A. Cardou, L. Cloutier, Strain measurements on ACSR conductors during fatigue tests II - Stress fatigue indicators, IEEE Trans. Power Deliv. 25, 2997-3006 (2010)
22. S. Goudreau, F. Lévesque, A. Cardou, L. Cloutier, Strain measurements on ACSR conductors during fatigue tests III - Strains related to support geometry, IEEE Trans. Power Deliv. 25, 3007-3016 (2010)

23. M.H. Maitournam, K. Dang Van, J.-F. Flavenot, Fatigue design of notched components with stress gradients and cyclic plasticity, Adv. Eng. Mater. 11, 750-754 (2009)

24. B. Crossland, Effect of large hydrostatic pressures on the torsional fatigue strength of an alloy steel, in: Proceedings of the International Conference on Fatigue of Metals, Institution of Mechanical Engineers, London, 1956, pp. 138-149

25. K.N. Smith, P. Watson, T.H. Topper, A stress-strain function for the fatigue of metals, J. Mater. 5, 767-778 (1970)

26. C. Ruiz, P.H.B. Boddington, K.C. Chen, An investigation of fatigue and fretting in a dovetail joint, Exp. Mech. 24, 208 217 (1984)

27. J. Redford, M.C. Nguyen, H.-P. Lieurade, M. Gueguin, F. Hafid, C. Yang, J.-M. Ghidaglia, Prediction of crack initiation due to fretting-fatigue in overhead power lines, Article en préparation

Citation de l'article : John Redford, Henri-Paul Lieurade, Maxime Gueguin, Fikri Hafid, Christine Yang, Jean-Michel Ghidaglia, Modélisation numérique du phénomène de fretting-fatigue intervenant dans le vieillissement des conducteurs de lignes aériennes, Matériaux \& Techniques 106, 308 (2018) 